\title{
Análisis comparativo en losas de cimentación: losas aligeradas mediante bloques de EPS frente a losas macizas
}

\section{Comparative analysis of slabs foundation: lightweight by expanded polystyrene blocks opposite solid slabs}

$\underline{\text { J. M. Dávila }}^{(*)}$, J. C. Fortes ${ }^{(*)}$, L. Crespo ${ }^{(*)}$, C. A. Rodríguez ${ }^{(*)}$

\section{RESUMEN}

El objetivo del presente artículo es confirmar el distinto comportamiento que presenta la solución tradicional de losa maciza frente a una aligerada, haciendo un análisis comparativo, aplicando el M.E.F., de la primera solución con una losa aligerada empleando bloques de poliestireno expandido (EPS) como material aligerante, uniéndose las zonas macizas bajo pilares mediante nervios. Se mantienen, por tanto, dos placas superior e inferior alrededor de los bloques aligerantes de EPS.

Los resultados obtenidos nos permiten afirmar que en situaciones de suelos blandos, con posibles problemas de asientos, la solución planteada presenta importantes ventajas, al disminuir éstos en un porcentaje que variará en función de las condiciones particulares. Además de esto, se reducen en gran medida los asientos diferenciales entre apoyos, por lo que la alternativa planteada presenta un comportamiento más uniforme, así como ventajas constructivas.

Palabras clave: Losa aligerada de cimentación; poliestireno expandido; elementos finitos; asientos; material aligerante; asientos diferenciales.

\section{ABSTRACT}

The objective of this article is to confirm the different behavior of the traditional slab foundation against a lightened, making a comparative analysis, using the MEF, of the first solution with a lightened slab using expanded polystyrene (EPS) blocks, joining solid areas on pillars with grating beams. Therefore there are two top and bottom plates covering the EPS blocks.

The results obtained allow assert ourselves that in situations of soft soils, with possible problems of settlements, the proposed solution has significant advantages by reducing them a percentage will vary depending on specific conditions. Besides this, it reduces significantly the differential settlements between supports, so this alternative has a more uniform behavior, as well as constructive advantages.

Keywords: Lightened slab foundation; expanded polystyrene; finite elements; settlements; lightening material; differential settlements.

(*) Universidad de Huelva. La Rábida-Palos de la Frontera, Huelva (España).

Persona de contacto/Corresponding author: jmdavila@dimme.uhu.es (J. M. Dávila)

Cómo citar este artículo/Citation: Dávila, J. M., Fortes, J. C., Crespo, L., Rodríguez, C. A. (2015). Análisis comparativo en losas de cimentación: losas aligeradas mediante bloques de EPS frente a losas macizas. Informes de la Construcción, 67(538): eo8o, doi: http://dx.doi.org/10.3989/ic.13.157.

Licencia/License: Salvo indicación contraria, todos los contenidos de la edición electrónica de Informes de la Construcción se distribuyen bajo una licencia de uso y distribución Creative Commons Reconocimiento no Comercial 3.o. España (cc-by-nc). 


\section{INTRODUCCIÓN}

Como alternativa a la losa maciza de hormigón armado, han surgido en las últimas décadas distintas aplicaciones, como la empleada parcialmente en la cimentación de La Sagrada Familia (Fachada de la Pasión), con la utilización de perfiles de acero a modo de emparrillado embebido en el canto de la losa (1), aun cuando en la práctica funcionaban como armaduras en tracción.

Tras la aparición del EPS (EPS Geofoam en su denominación en inglés $)^{1}$ y su posterior aplicación como material de construcción, han sido muchos los campos a los que se ha dirigido su empleo, siendo uno de ellos el de la geotecnia. Existen estudios recientes en este campo (2) (3) en los que se han analizado sus propiedades y posibles aplicaciones; destacando su empleo como material de relleno, incluso como árido en forma de bolas para morteros ligeros (4), y otras aprovechando la ventaja deducida de su baja densidad.

Recientemente, ha aparecido en el mercado, una solución constructiva denominada Cimentur (5) en la que mediante el empleo de bloques de EPS se puede realizar un vaciado completo de la superficie ocupada por la cimentación. Esta solución permite una ejecución más rápida; sin embargo, conceptualmente, no supone aportación alguna desde un punto de vista puramente estructural o geotécnico, entendiéndola como una mejora constructiva.

\footnotetext{
${ }^{1}$ En este artículo se hace referencia al EPS como poliestireno expandido en bloques o elementos rígidos, existiendo en el mercado algunas resinas denominadas Geofoam que se emplean mediante inyección.
}

Una solución con cierta semejanza a la anterior (aplicable a viviendas unifamiliares) consiste en una losa apoyada sobre un continuo de bloques de poliestireno expandido, a la que se le añaden unos nervios en los bordes hormigonados sobre unas piezas prefabricadas de EPS (6).

\section{OBJETIVOS}

Planteamos en este trabajo una alternativa a la tradicional solución de cimentación mediante losa maciza. Ésta aparece recogida en distintas publicaciones (7). En estas soluciones el conjunto está concebido a modo de superestructura entre las losas y los muros de sótano, opción ya empleada asiduamente en edificios de gran altura. La solución que aquí se propone consistiría en un elemento único de cimentación mediante una losa aligerada, en la que estas zonas estarían «rellenas» a modo de encofrado perdido, con bloques de EPS. Mediante esta opción se pretenden obtener dos mejoras en la cimentación, tal como ya se apunta en algunas publicaciones (8): por un lado, un aumento de rigidez sin coste en volumen de hormigón; y por otro, reducir en lo posible los asientos.

Como objetivos concretos de esta investigación se plantean los siguientes:

1. Estudiar la posibilidad de uso de los bloques de EPS como piezas aligerantes en estructuras de cimentación.

2. Comparar la solución de losa maciza frente a la aligerada.

3. Analizar el comportamiento estructural de una losa aligerada con EPS.

4. Evaluar el comportamiento frente a asientos en este tipo de soluciones.

5. Evaluar la viabilidad técnica de la solución propuesta.

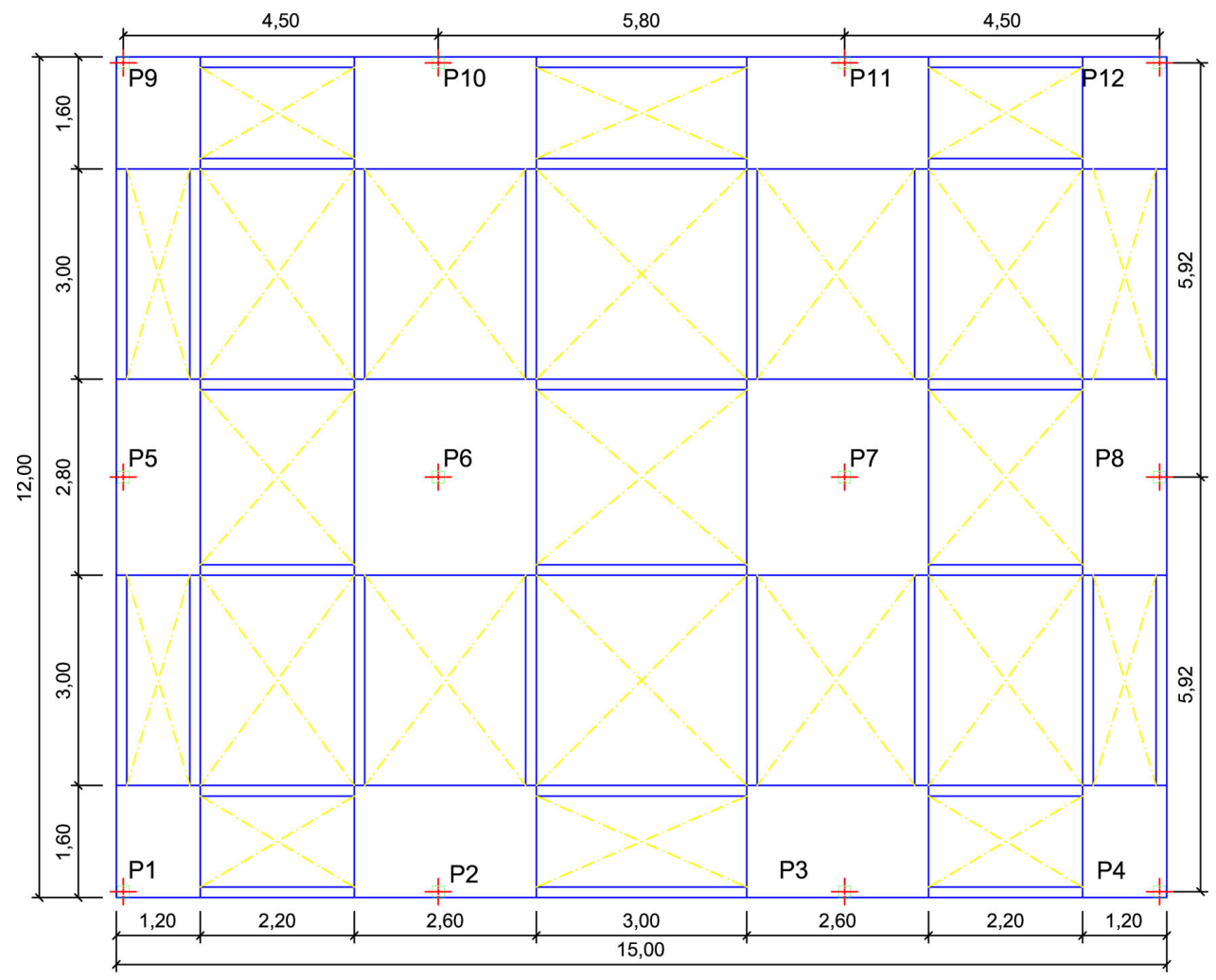

Figura 1. Esquema en planta de la cimentación estudiada. 15,00 × 12,00. 


\section{CARACTERÍSTICAS DEL MODELO}

Se han analizado dos soluciones distintas de losas aligeradas, con distintas dimensiones y estado de cargas. También se han estudiado para la losa de menores dimensiones, distintas condiciones en el terreno, comparándose los distintos casos con soluciones similares resueltas mediante losa maciza.

El primero de los casos estudiados es una losa de $8,10 \times$ 6,45 $\mathrm{m}$ y $50 \mathrm{~cm}$ de canto, comparada con una losa maciza del mismo canto, esquema típico de viviendas adosadas de dos plantas. El segundo caso se trata de una losa de 12,00 $\times$ 15,00 m y $70 \mathrm{~cm}$ de canto (Figura 1). En ambas situaciones en la losa se han dispuesto doce arranques de pilares, con zonas macizas en el área de los soportes y nervios arrancando de las esquinas de éstas. También en los dos esquemas la geometría consta de sendas losas superior e inferior de $10 \mathrm{~cm}$ cada una de ellas, quedando por tanto en el primer caso una zona aligerada de $30 \mathrm{~cm}$ a ocupar con los bloques de EPS, mientras que en la segunda la geometría ha sido también de dos losas extremas de $10 \mathrm{~cm}$, resultando una zona aligerada de $50 \mathrm{~cm}$. El primer caso nos permitirá evaluar la bondad de la solución en este tipo de edificaciones, mientras que con la segunda opción podremos analizar construcciones de mayores luces y con mayor número de plantas.

Las características de los materiales empleados se muestran en la Tabla 1.

Los coeficientes de ponderación de acciones contemplados son:

Permanentes $\gamma_{\mathrm{g}}=1,35$

Variables $\gamma_{\mathrm{f}}=1,50$

Tabla 1. Características de los materiales empleados (programa SAP200o).

\begin{tabular}{|l|c|l|}
\hline & Hormigón HA-25 & $\begin{array}{c}\text { Poliestireno } \\
\text { expandido* }\end{array}$ \\
\hline Módulo de elasticidad & $\mathrm{E}=2,5 \cdot 10^{4} \mathrm{MPa}$ & $\mathrm{E}=6 \mathrm{MPa}$ \\
\hline Coeficiente de Poisson & $\mathrm{v}=0,2$ & $\mathrm{v}=0,1$ \\
\hline Peso específico & $\gamma=24 \mathrm{kN} / \mathrm{m}^{3}$ & $\gamma=0,3 \mathrm{kN} / \mathrm{m}^{3}$ \\
\hline $\begin{array}{l}\text { Coeficiente de balasto } \\
\text { inicial }\end{array}$ & \multicolumn{2}{|c|}{$1000 \mathrm{kN} / \mathrm{m}^{3}$} \\
\hline
\end{tabular}

* Es importante señalar, que los valores del Módulo de deformación longitudinal recogidos aquí se corresponden con los aportados por los distintos fabricantes, y contemplados, así mismo, por la normativa que es de aplicación. Sin embargo, se ha demostrado (9) que en piezas de gran tamaño, este valor es bajo.
En la menor de las dos losas estudiadas el sistema de cargas aplicado ha sido el siguiente: (Tabla 2).

Estos valores de cargas corresponden a un esquema típico de un edificio de viviendas de dos plantas.

Un esquema similar a éste es el que se ha empleado en el segundo de los casos analizados, con una losa de mayores dimensiones y acciones. Las cargas que se han aplicado aquí se muestran en la Tabla 3 .

Aun cuando existen alternativas al modelo de Winkler (10), entendemos que éste nos da una aproximación válida para nuestro propósito, más aún, cuando pretendemos hacer una análisis comparativo entre la solución de placa maciza y la aligerada. Para analizar estas cimentaciones hemos empleado en primer lugar, el programa de elementos finitos SAP 2000 (versión 14), apoyándonos en estudios comparativos de métodos analíticos, numéricos y el MEF (11) (12); en el que se han utilizado elementos tipo shell de cuatro nodos y $10 \mathrm{~cm}$ de espesor para las placas superior e inferior (el subtipo Shell contempla un comportamiento conjunto tipo placa y tipo membrana); elementos shell de 15 y $20 \mathrm{~cm}$ para los nervios de la losa menor y mayor respectivamente. Para las zonas macizas se han empleado, así mismo, elementos tipo placa en ambas direcciones. En todos los casos se ha aplicado la opción «Shell-Thin» de placas de pequeño espesor. El esquema de la modelización se puede ver en la siguiente Figura 2.

Con esta primera fase pretendemos hacer un análisis estructural de la solución propuesta, mientras que en una segunda fase, empleando el programa Plaxis 3D Túnel, se estudiaría su comportamiento geotécnico. Aquí se han empleado elementos finitos tridimensionales prismáticos de 14 nodos.

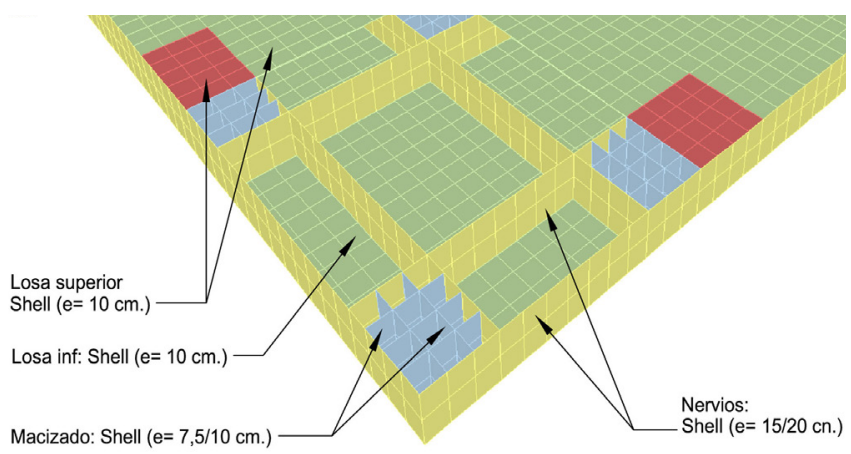

Figura 2. Esquema del modelo con los elementos empleados.

Tabla 2. Sistema de cargas aplicado (losa menor).

\begin{tabular}{|l|c|c|c|c|}
\hline & $\begin{array}{c}\text { Pilares de esquina } \\
\text { P1, P4, P9 y P12 }\end{array}$ & $\begin{array}{c}\text { Pilares de borde } \\
\text { P2, P3, P10 y P11 }\end{array}$ & $\begin{array}{c}\text { Pilares de borde } \\
\text { P5 y P8 }\end{array}$ & $\begin{array}{c}\text { Pilares interiores } \\
\text { P6 y P7 }\end{array}$ \\
\hline Permanentes & $45 \mathrm{kN}$ & $70 \mathrm{kN}$ & $65 \mathrm{kN}$ & $40 \mathrm{kN}$ \\
\hline Variables & $10 \mathrm{kN}$ & $20 \mathrm{kN}$ & $17 \mathrm{kN}$ & $10 \mathrm{kN}$ \\
\hline
\end{tabular}

Tabla 3. Sistema de cargas aplicado (losa mayor).

\begin{tabular}{|l|c|c|c|c|}
\hline & $\begin{array}{c}\text { Pilares de esquina } \\
\text { P1, P4, P9 y P12 }\end{array}$ & $\begin{array}{c}\text { Pilares de borde } \\
\text { P2, P3, P10 y P11 }\end{array}$ & $\begin{array}{c}\text { Pilares de borde } \\
\text { P5 y P8 }\end{array}$ & $\begin{array}{c}\text { Pilares interiores } \\
\text { P6 y P7 }\end{array}$ \\
\hline Permanentes & $300 \mathrm{kN}$ & $440 \mathrm{kN}$ & $400 \mathrm{kN}$ & $600 \mathrm{kN}$ \\
\hline Variables & $90 \mathrm{kN}$ & $120 \mathrm{kN}$ & $100 \mathrm{kN}$ & $240 \mathrm{kN}$ \\
\hline
\end{tabular}


Para modelizar la losa en el programa Plaxis 3D se han empleado los siguientes datos: (Tabla 4).

No se considera nivel freático.

El modelo discretizado quedaría tal como aparece en la Figura 3 (se ha modelado únicamente la mitad de la losa).

\section{RESULTADOS OBTENIDOS}

Tanto la losa menor como la de mayores dimensiones, se han estudiado para diferentes valores del coeficiente de balasto, recogiéndose los resultados en las Tablas 5 y 6 . Análogamente, se recogen los valores de asientos máximos y el porcentaje de reducción de éstos a partir del modelo realizado mediante el programa Plaxis (Tabla 7).

Tabla 4. Datos empleados (programa Plaxis 3D).

\begin{tabular}{|l|l|l|l|l|}
\hline & \multicolumn{1}{|c|}{$\begin{array}{c}\text { Hormigón } \\
\text { HA-25 }\end{array}$} & $\begin{array}{c}\text { Poliestireno } \\
\text { expandido }\end{array}$ & $\begin{array}{c}\text { Terreno nivel 1 } \\
\text { (8 m) } \\
\text { arcillas blandas }\end{array}$ & $\begin{array}{c}\text { Terreno nivel } 2 \\
\text { (10 m) } \\
\text { arena media }\end{array}$ \\
\hline Módulo de elasticidad & $\mathrm{E}=2,5 \cdot 10^{4} \mathrm{MPa}$ & $\mathrm{E}=6 \mathrm{MPa}$ & $\mathrm{E}=5 \mathrm{MPa}$ & $\mathrm{E}=10 \mathrm{MPa}$ \\
\hline Coeficiente de Poisson & $\mathrm{v}=0,2$ & $\mathrm{v}=0,1$ & $\mathrm{v}=0,32$ & $\mathrm{v}=0,3$ \\
\hline Peso específico & $\mathrm{\gamma}=24 \mathrm{kN} / \mathrm{m}^{3}$ & $\gamma=0,3 \mathrm{kN} / \mathrm{m}^{3}$ & ${ }^{*} \gamma_{\mathrm{d}}=17 \mathrm{kN} / \mathrm{m}^{3}$ & ${ }^{*} \gamma_{\mathrm{d}}=18 \mathrm{kN} / \mathrm{m}^{3}$ \\
\hline Cohesión & - & - & $\mathrm{c}=5 \mathrm{kN} / \mathrm{m}^{2}$ & $\mathrm{c}=1 \mathrm{kN} / \mathrm{m}^{2}$ \\
\hline $\begin{array}{l}\text { Ángulo de rozamiento } \\
\text { interno }\end{array}$ & \multicolumn{1}{|c}{-} & $\vartheta=25^{\circ}$ & $\vartheta=30^{\circ}$ \\
\hline
\end{tabular}

* Peso específico aparente

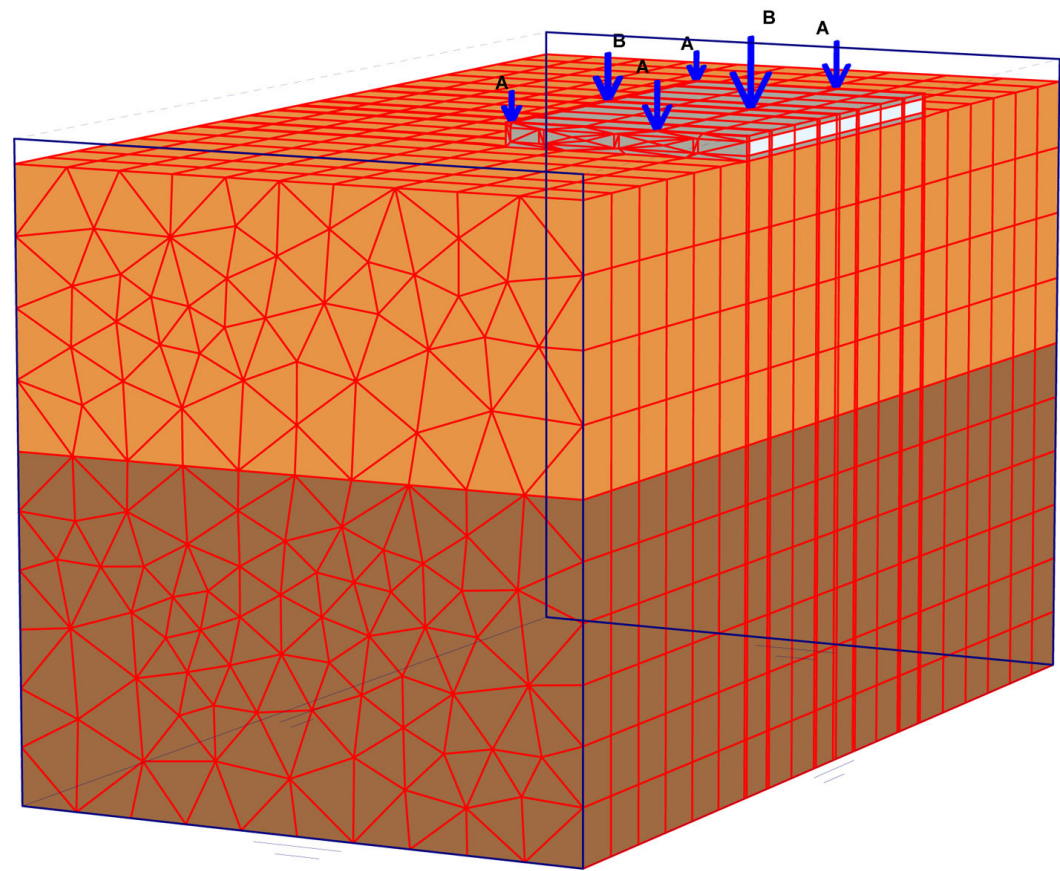

Figura 3. Modelo 3D de la losa en Plaxis 3D.

Tabla 5. Resultados obtenidos en la losa 8,10 × 6,45 (acciones características).

\begin{tabular}{|c|c|c|c|c|c|c|c|c|c|c|c|c|c|c|c|c|}
\hline$K_{\text {losa }}\left(k N / \mathbf{m}^{3}\right)$ & \multicolumn{4}{|c|}{$K_{\text {losa }}=1.000$} & \multicolumn{4}{|c|}{$K_{\text {losa }}=2.000$} & \multicolumn{4}{|c|}{$K_{\text {losa }}=5.000$} & \multicolumn{4}{|c|}{$K_{\text {losa }}=10.000$} \\
\hline $\begin{array}{l}\text { ASIENTOS } \\
(\mathrm{mm} .)\end{array}$ & $\mathrm{S}_{\max }$ & $\mathrm{S}_{\min }$ & $\mathrm{S}_{\text {medio }}$ & $\begin{array}{c}\text { Diferencia } \\
\mathrm{S}_{\max } \mathrm{S}_{\min }(\%)\end{array}$ & $\mathrm{S}_{\max }$ & $\mathrm{S}_{\min }$ & \begin{tabular}{|l|}
$\mathrm{S}_{\text {medio }}$ \\
\end{tabular} & $\begin{array}{c}\text { Diferencia } \\
\mathrm{S}_{\max }, \mathrm{S}_{\min }(\%)\end{array}$ & $\mathrm{S}_{\max }$ & $\mathrm{S}_{\min }$ & $\mathrm{S}_{\text {medio }}$ & $\begin{array}{c}\text { Diferencia } \\
\mathrm{S}_{\max ,} \mathrm{S}_{\min }(\%)\end{array}$ & $\mathrm{S}_{\max }$ & $\mathrm{S}_{\min }$ & $\mathrm{S}_{\text {medio }}$ & \begin{tabular}{|c|} 
Diferencia \\
$\mathrm{S}_{\max } \mathrm{S}_{\min }(\%)$
\end{tabular} \\
\hline MACIZA & 50,3 & 48,4 & 49,4 & 3,8 & 25,7 & 23,9 & 24,8 & 7,0 & 10,9 & 9,2 & 10,1 & 15,6 & 5,9 & 4,4 & 5,2 & 25,4 \\
\hline ALIGERADA & 49,2 & 48,2 & 48,7 & 2,0 & 24,9 & 24,2 & 24,6 & 2,8 & 9,9 & 9,5 & 9,7 & 4,0 & 5,0 & 4,6 & 4,8 & 8,0 \\
\hline
\end{tabular}

Tabla 6. Resultados obtenidos en la losa $15 \times 12$. Solución (1) mediante elementos tipo Shell y (2) mediante elementos Solid (acciones características).

\begin{tabular}{|c|c|c|c|c|c|c|c|c|c|c|c|c|c|c|c|c|}
\hline$K_{\text {losa }}\left(\mathrm{kN} / \mathrm{m}^{3}\right)$ & \multicolumn{4}{|c|}{$K_{\text {losa }}=1.000$} & \multicolumn{4}{|c|}{$K_{\text {losa }}=2.000$} & \multicolumn{4}{|c|}{$K_{\text {losa }}=5.000$} & \multicolumn{4}{|c|}{$K_{\text {losa }}=10.000$} \\
\hline $\begin{array}{l}\text { ASIENTOS } \\
(\mathrm{mm} .)\end{array}$ & $\mathrm{S}_{\max }$ & $\mathrm{S}_{\min }$ & $\mathrm{S}_{\text {medio }}$ & $\begin{array}{c}\text { Diferencia } \\
\mathrm{S}_{\max } \mathrm{S}_{\min }(\%)\end{array}$ & $\mathrm{S}_{\max }$ & $\mathrm{S}_{\min }$ & $\mathrm{S}_{\text {medio }}$ & $\begin{array}{c}\text { Diferencia } \\
\mathrm{S}_{\max } \mathrm{S}_{\min }(\%)\end{array}$ & $\mathrm{S}_{\text {max }}$ & $\mathrm{S}_{\min }$ & $\mathrm{S}_{\text {medio }}$ & $\begin{array}{c}\text { Diferencia } \\
\mathrm{S}_{\max ,} \mathrm{S}_{\min }(\%)\end{array}$ & $\mathrm{S}_{\max }$ & $\mathrm{S}_{\min }$ & $\mathrm{S}_{\text {medio }}$ & $\begin{array}{c}\text { Diferencia } \\
\mathrm{S}_{\max } \mathrm{S}_{\min }(\%)\end{array}$ \\
\hline MACIZA & 59,5 & 51,6 & 55,6 & 13,3 & 32,4 & 24,6 & 28,5 & 24,1 & 14,9 & 8,8 & 11,9 & 40,9 & 8,8 & 3,9 & 6,4 & 55,7 \\
\hline $\begin{array}{l}\text { ALIGERADA } \\
\text { (1) }\end{array}$ & 47,3 & 44,2 & 45,8 & 6,6 & 23,9 & 20,9 & 22,4 & 12,6 & 9,9 & 7,2 & 8,6 & 27,3 & 5,2 & 2,8 & 4,0 & 46,2 \\
\hline $\begin{array}{l}\text { ALIGERADA } \\
\text { (2) }\end{array}$ & 47,8 & 45,5 & 46,7 & 4,8 & 24,3 & 21,8 & 23,1 & 10,3 & 10,1 & 7,7 & 8,9 & 23,8 & 5,3 & 3,1 & 4,2 & 41,5 \\
\hline
\end{tabular}


Tabla 7. Resultados comparativos de asientos obtenidos con Plaxis.

\begin{tabular}{|c|c|c|c|c|c|c|c|c|}
\hline \multirow{2}{*}{.E (MPa) } & \multicolumn{2}{|c|}{$\mathbf{E}_{\mathrm{coh}}=5$} & \multicolumn{2}{|c|}{$E_{\text {coh }}=10$} & \multicolumn{2}{|c|}{$\mathbf{E}_{\text {coh }}=\mathbf{2 0}$} & \multicolumn{2}{|c|}{$\mathrm{E}_{\text {coh. }}=\mathbf{3 0}$} \\
\hline & \multicolumn{2}{|c|}{$\mathrm{E}_{\text {gran. }} 10$} & \multicolumn{2}{|c|}{$\mathbf{E}_{\text {gran. }} 20$} & \multicolumn{2}{|c|}{$\mathrm{E}_{\text {gran. }} 30$} & \multicolumn{2}{|c|}{$\mathrm{E}_{\text {gran. }} 40$} \\
\hline $\begin{array}{l}\text { ASIENTOS } \\
(\mathrm{mm})\end{array}$ & $\mathrm{S}_{\max }$ & $\begin{array}{c}\text { Reducción } \\
\mathrm{S}_{\max }(\%)\end{array}$ & $\mathrm{S}_{\max }$ & $\begin{array}{c}\text { Reducción } \\
\mathrm{S}_{\max }(\%)\end{array}$ & $\mathrm{S}_{\max }$ & $\begin{array}{c}\text { Reducción } \\
\mathrm{S}_{\max }(\%)\end{array}$ & $\mathrm{S}_{\max }$ & $\begin{array}{c}\text { Reducción } \\
\mathrm{S}_{\max }(\%)\end{array}$ \\
\hline MACIZA & 59,2 & \multirow{2}{*}{13,4} & 29,9 & \multirow{2}{*}{12,8} & 14,8 & \multirow{2}{*}{12,2} & 10,3 & \multirow{2}{*}{12,0} \\
\hline ALIGERADA & 51,3 & & 26,1 & & 13,0 & & 9,1 & \\
\hline
\end{tabular}

Se presentan a continuación los resultados obtenidos con Sap20oo en cuanto a deformaciones, tanto en la losa maciza, como en la aligerada (Figura 4).

De la misma forma, incluimos también los resultados obtenidos en la losa de $12 \times 15 \mathrm{~m}$ (Figura 5). En todos los gráficos presentados, aparecen los resultados para la combinación de acciones permanentes junto a las acciones variables.

\section{ANÁlISIS DE RESULTADOS}

Siguiendo el mismo orden que en el precedente apartado, pasamos a analizar los resultados obtenidos en los distintos modelos realizados.

En lo referente al modelo de dimensiones menores, la diferencia en cuanto a los valores máximos de asientos es mínima (entre 66,6 y 68,9 mm para la solución de losa maciza; y entre 66,5 y $67,1 \mathrm{~mm}$ en la aligerada, ambos en la combinación de acciones permanentes y variables), lo que por otra parte era de esperar, dada la escasa repercusión de la zona aligerada en las cargas totales; pero sin embargo, hay importantes diferencias en cuanto a la distribución de las deformaciones: en la solución adoptada de losa aligerada, se minimiza el efecto de asientos en bordes, y además se consigue una distribución de asientos mucho más regular a lo largo de toda la superficie de la losa, como se comprueba en la distribución de los tonos verdes de la Figura 4.

En el segundo modelo estudiado, se observa (Figura 5) como la diferencia en asientos máximos para las soluciones maciza y aligerada ha crecido de forma apreciable $(71,5 \mathrm{~mm}$ frente a $62 \mathrm{~mm}$, estando ahora esta diferencia cercana al $20 \%$ para los valores característicos). Además de ésta, la otra diferencia
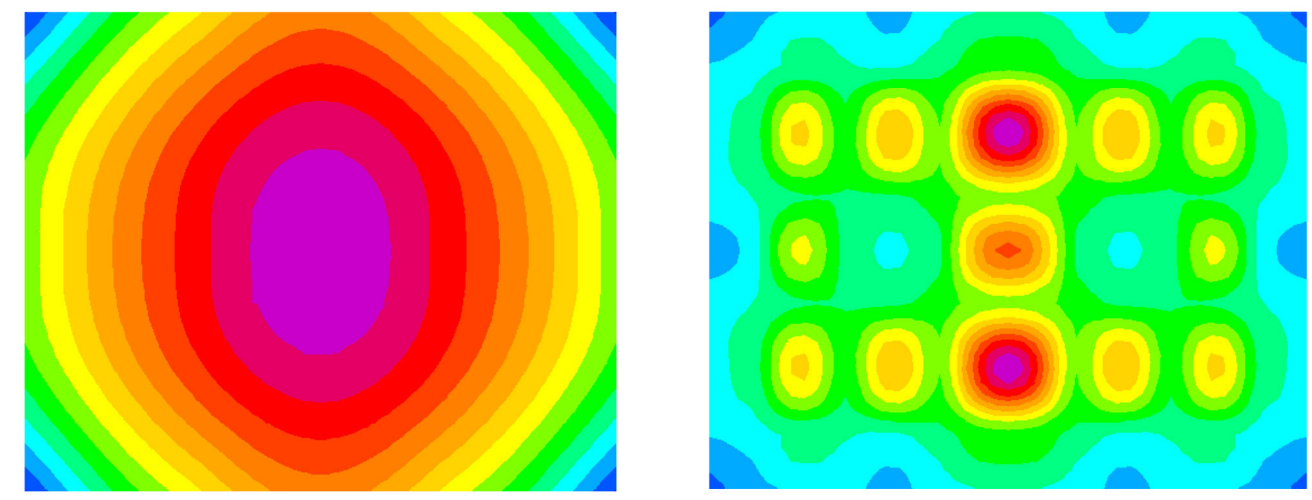

Figura 4. Gráfico de asientos. Izq.: Losa maciza 8,10 × 6,45 (asiento máximo con acciones características $51 \mathrm{~mm}$ ). Drcha.: Losa aligerada 8,10 × 6,45 (asiento máximo con acciones características $49 \mathrm{~mm}$ ).
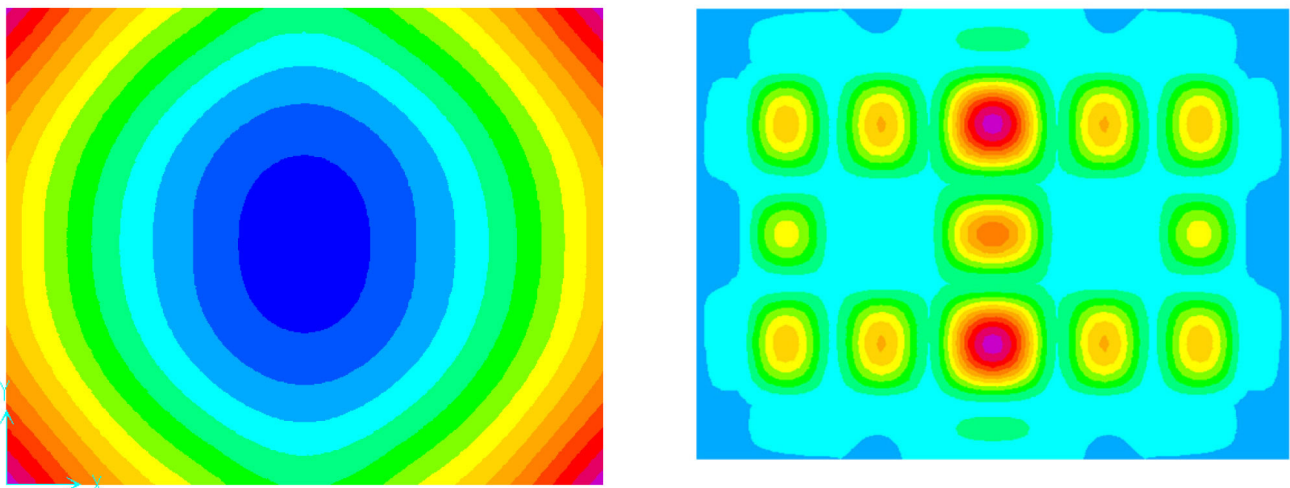

Figura 5. Gráfico de asientos. Izq.: Losa maciza $12 \times 15$ (asiento máximo con acciones características 59 mm). Drcha.: Losa aligerada $12 \times 15$ (asiento máximo con acciones características $48 \mathrm{~mm}$ ). 
destacable en este caso, es que los asientos menores se concentran algo más en los vanos que en el caso anterior.

En la Figura 6, aparecen de forma comparativa los resultados de la losa aligerada frente a la maciza, en la menor de las losas, mientras que estos mismos, son los reflejados en la Figura 7 para la losa mayor.

Tal como se apuntó en párrafos anteriores, se puede apreciar claramente en las Figuras 6 y 7, como las diferencias entre la solución aligerada y la maciza, son mucho más significativas en la losa con acciones superiores y mayor longitud de vano. Por esa razón, se decidió comparar, así mismo, los resultados de la losa aligerada, modelándola, por un lado, con elementos tipo Shell, y por otro, con elementos tipo Solid en las zonas macizas.

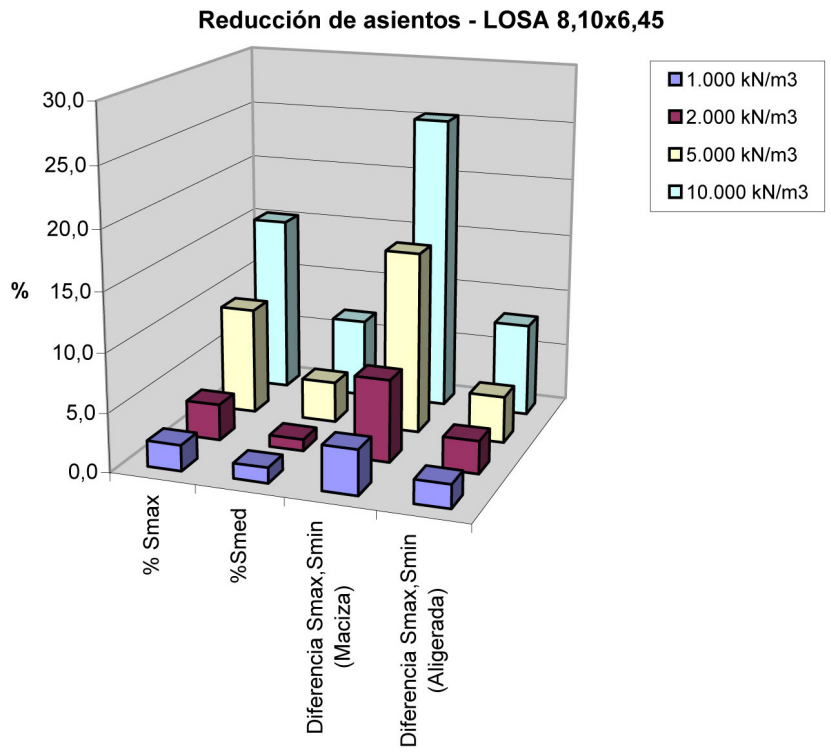

Figura 6. Comparativa losa maciza - aligerada (losa 8,10 × 6,45).
A la vista de la Figura 7, si se observan los porcentajes de reducción de asientos máximos y medios, podemos también concluir, que además de ser significativa la reducción de los máximos, es aún más relevante la reducción de asientos diferenciales, lo que se puede confirmar analizando las dos últimas columnas de cada figura, en la que aportamos los valores de la diferencia entre asientos máximos y mínimos en porcentaje (en torno a L/100o para la losa maciza y L/300oo para la aligerada, como distorsiones máximas entre pilares).

Si se analizan los resultados obtenidos para la solución de la placa aligerada, con los dos modelos empleados (todo el modelo con elementos shell, frente a un modelo con las zonas macizas mediante elementos solid), se aprecia cómo siendo los valores de asientos máximos muy similares para ambas soluciones, en el caso de emplearse elementos sólidos, se obtienen unos asientos mínimos ligeramente superiores, y por tanto, asientos diferenciales menores. En cualquier caso, estas diferencias entre ambos modelos no parecen demasiado relevantes, por lo que, en la práctica, puede emplearse cualquiera de los modelos obteniéndose soluciones igualmente válidas.

Junto al análisis previo de los asientos en ambas placas, se presentan a continuación los resultados para los esfuerzos de flexión obtenidos en la mayor de las dos placas, comparando la solución maciza y la aligerada que aquí se plantea. Si se comparan las tensiones normales obtenidas para las dos soluciones en la zona inferior de las losas, se pueden obtener importantes conclusiones: mientras que en la losa maciza, aparecen claramente marcadas las bandas de soportes y bandas centrales (Figura 8), en el caso de la losa aligerada, las tensiones marcan la situación de los nervios, como elementos fundamentales en la flexión (Figura 9). Por lo tanto, es en esas zonas, donde aparecen las máximas tensiones $\left(3,80 \mathrm{~N} / \mathrm{mm}^{2}\right.$ en la maciza y 17,18 en la aligerada). En el caso de la losa aligerada, se obtienen para ambas tensiones, valores muy bajos en los bordes -
Reducción de asientos - LOSA 15x12 (Shell)

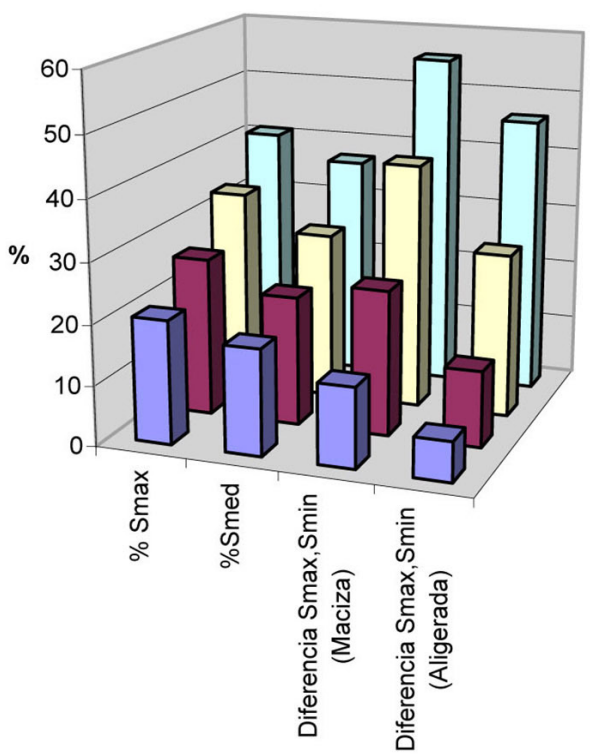

Reducción de asientos - LOSA 15x12 (Solid)

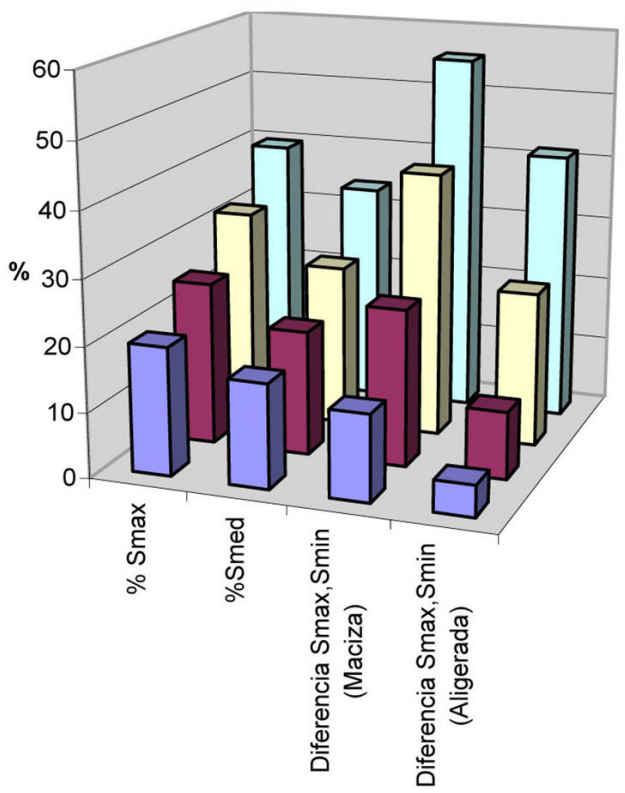

口 $1.000 \mathrm{kN} / \mathrm{m} 3$ 口2.000 kN/m3 口 $5.000 \mathrm{kN} / \mathrm{m} 3$ 口 $10.000 \mathrm{kN} / \mathrm{m} 3$

Figura 7. Comparativa losa maciza - aligerada (losa $15 \times 12)$. 


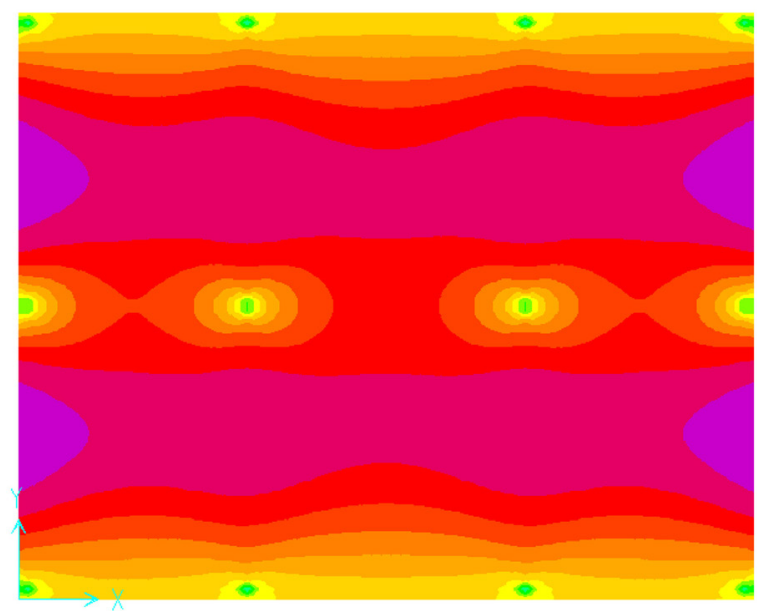

Figura 8. Distribución de tensiones $\mathrm{S}_{22}$ en la losa maciza. (Combinación A. Permanentes + A. Variables).

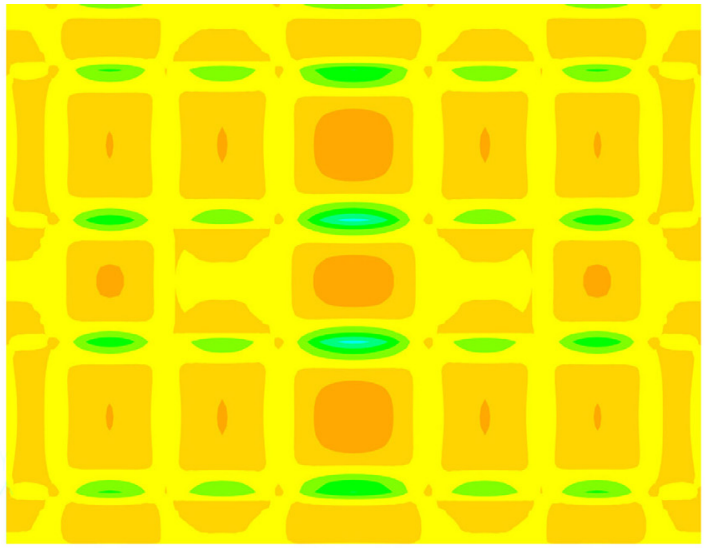

\begin{tabular}{llllllllllllll}
$-24,0$ & $-20,0$ & $-16,0$ & $-12,0$ & $-8,0$ & $-4,0$ & 0,0 & 4,0 & 8,0 & 12,0 & 16,0 & 20,0 & 24,0 & 28,0 \\
\hline
\end{tabular}

Figura 9. Distribución de tensiones $\mathrm{s}_{22}$ en la placa inferior de la losa aligerada. (Combinación A. Permanentes + A. Variables). tanto transversal como longitudinalmente - e incluso en gran parte de las zonas de vanos, lo que supondrá un armado por cuantía mínima, salvo lógicamente, en las zonas ocupadas por los nervios. Por el contrario, es en las bandas de soportes exteriores (longitudinal y transversal) donde se obtendrían las tensiones (y momentos) máximos en la losa maciza.

Se han representado en la figuras 10 las leyes de momentos flectores de las dos soluciones. Del análisis de ambos gráficos, se pueden extraer algunas ideas fundamentales. Si se observan los valores máximos, tanto positivos como negativos, es claro, que en el caso de la solución aligerada, éstos se mantienen casi constantes entre los distintos vanos, mientras que en la losa maciza hay importantes diferencias entre los vanos exteriores y el vano central. Además de esto, en el diagrama de la losa maciza, las leyes siguen una distribución aproximadamente parabólica, Por el contrario, en la propuesta de la losa aligerada, se pueden apreciar zonas ascendentes y descendentes casi lineales, junto a las zonas de negativos con «lomas» bastante extendidas en longitud en los vanos, coincidiendo dichas zonas ascendentes - descendentes con las zonas macizas.

Si pasamos ahora a estudiar la siguiente Figura 11, correspondientes al primer nervio longitudinal interior en el caso de la losa aligerada (cota $y=+0,80 \mathrm{~m}$ ), se aprecia como las «lomas» y zonas ascendente - descendentes permanecen, pero menos acentuadas que en el caso del nervio de borde; sin embargo, se siguen manteniendo unos valores máximos (tanto positivos como negativos) muy similares entre vanos.

Para analizar el comportamiento de los nervios, se han comparado además, con el programa SAP2000, los resultados obtenidos en la menor de las dos losas, con una solución en la que eliminamos las losas superior e inferior, por tanto se convierte en una solución que podríamos denominar combinada zapata-emparrillado (obsérvese la Figura 15).
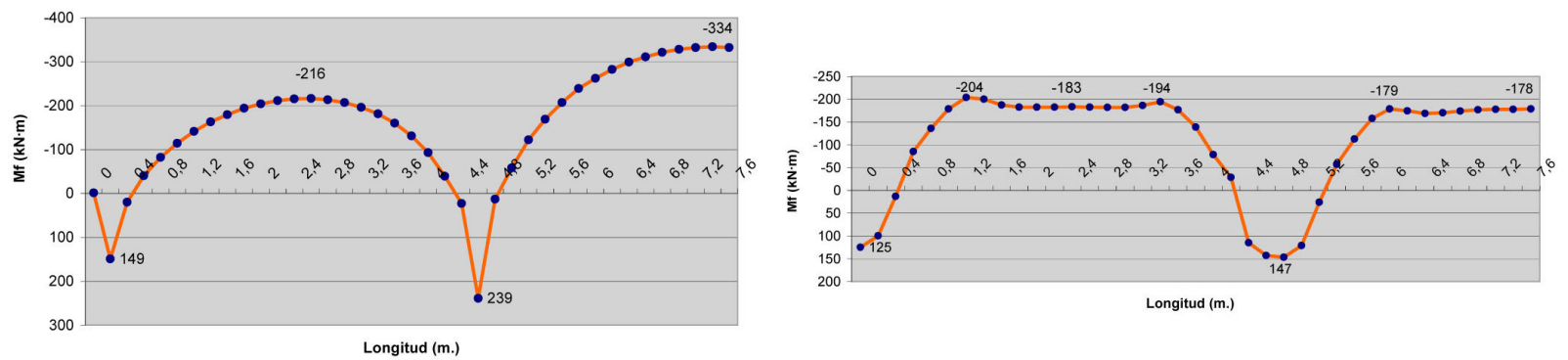

Figura 10. Ley de momentos en borde longitudinal (solo se representa la mitad). Izq.: Banda de soportes de borde de losa maciza. Drcha. Nervio de borde de losa aligerada.
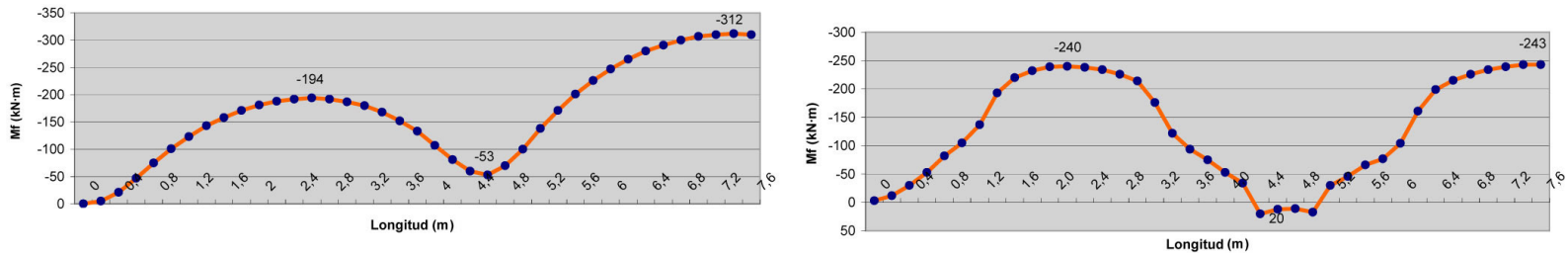

Figura 11. Ley de momentos en línea interior longitudinal (solo se representa la mitad). Izq.: Línea interior ( $\mathrm{y}=+0,80 \mathrm{~m})$ de losa maciza. Drcha.: Nervio interior de losa aligerada. 
Se ha podido constatar que en los dos esquemas el mecanismo estructural es, lógicamente distinto. En las Figuras 12 y 13 , presentadas a continuación, se reflejan los valores de las componentes normales $\mathrm{F}_{11}$ entendiéndose, que mientras que en el modelo de la Figura 15 la misión de los nervios es básicamente de atado entre los macizos, en el modelo aligerado, los nervios contribuyen a la flexión de la placa, a modo de «vigas centradoras» entre zonas macizas exteriores e interiores. Como se podía esperar, los resultados en cuanto a asientos no son comparables tanto en lo que se refiere a valores máximos (49 y $51 \mathrm{~mm}$ respectivamente para losa aligerada y maciza, frente a los 53,4 obtenidos aquí en los dos soportes interiores) como a su distribución en los distintos apoyos de soportes (los coeficientes de balasto adoptados para cada una de las superficies de apoyo han sido 3.000, 4.400, 5.100 y $\left.5.000 \mathrm{kN} / \mathrm{m}^{3}\right)$.

Deteniéndonos ahora en los resultados alcanzados con Plaxis, el análisis comparativo de ambas soluciones nos indica que, si bien el comportamiento general es muy similar en las dos, lo asientos máximos obtenidos se reducen aproximada- mente en un $14 \%$ para la losa aligerada (frente a la diferencia obtenida mediante el programa SAP que era del $19 \%$ - ver Tabla 7). Esto unido a asientos más uniformes con la solución que incluye zonas aligeradas, la otorgan, desde un punto de vista técnico, como una solución superior a la tradicional. Así mismo, se confirma una ligera reducción, de la diferencia de asientos entre la solución maciza y aligerada, al reducirse estos.

En cuanto a la distribución de tensiones, como era de esperar, solo se aprecian ligeras diferencias entre ambas soluciones en las capas más superficiales (tonos azules en la Figura 16), pasando de $0,336 \mathrm{~N} / \mathrm{mm}^{2}$ para las tensiones verticales en la losa maciza, frente a $0,334 \mathrm{~N} / \mathrm{mm}^{2}$ en la aligerada; mientras que en las tensiones de corte (tonos azules y naranjas bajo los bordes en la Figura 17) los valores van de $0,336 \mathrm{~N} / \mathrm{mm}^{2}$ en la losa maciza a $0,334 \mathrm{~N} / \mathrm{mm}^{2}$ en la aligerada.

Independientemente del mecanismo de trabajo de la losa, entendemos que el sistema presentaría algunos problemas constructivos, como sería la sustentación del armado superior, o la

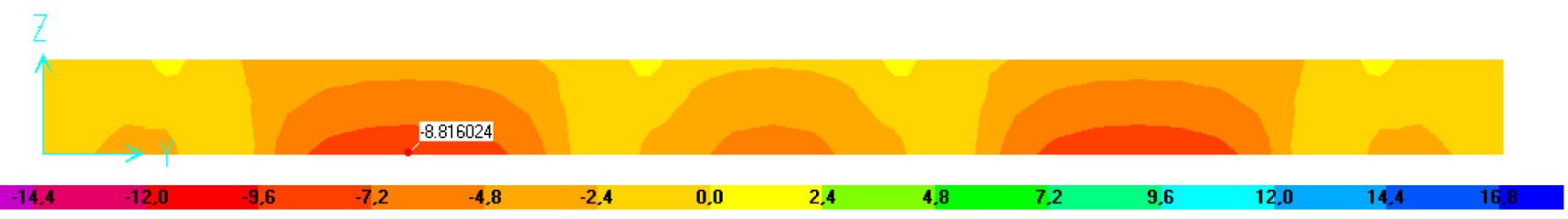

Figura 12 Componente $\mathrm{F}_{11}$ en nervio interior transversal de la primera línea de soportes. Losa aligerada. (Combinación Permanentes + Variables $-\mathrm{kN} / \mathrm{m}$ ).

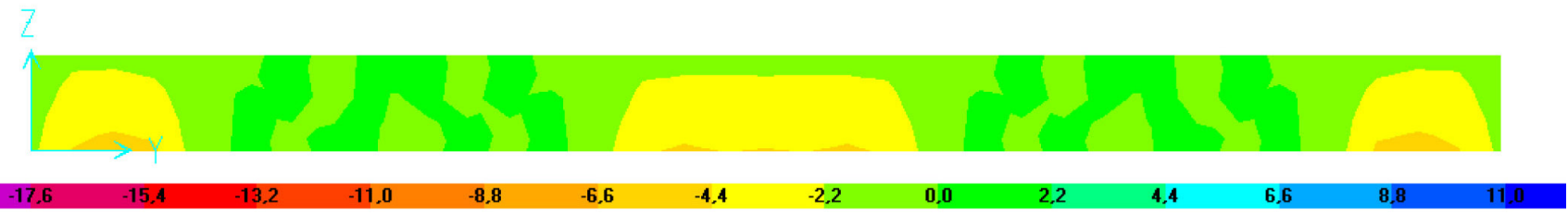

Figura 13. Componente $\mathrm{F}_{11}$ en nervio interior. Zapata-Emparrillado. (Combinación Permanentes + Variables - kN/m).

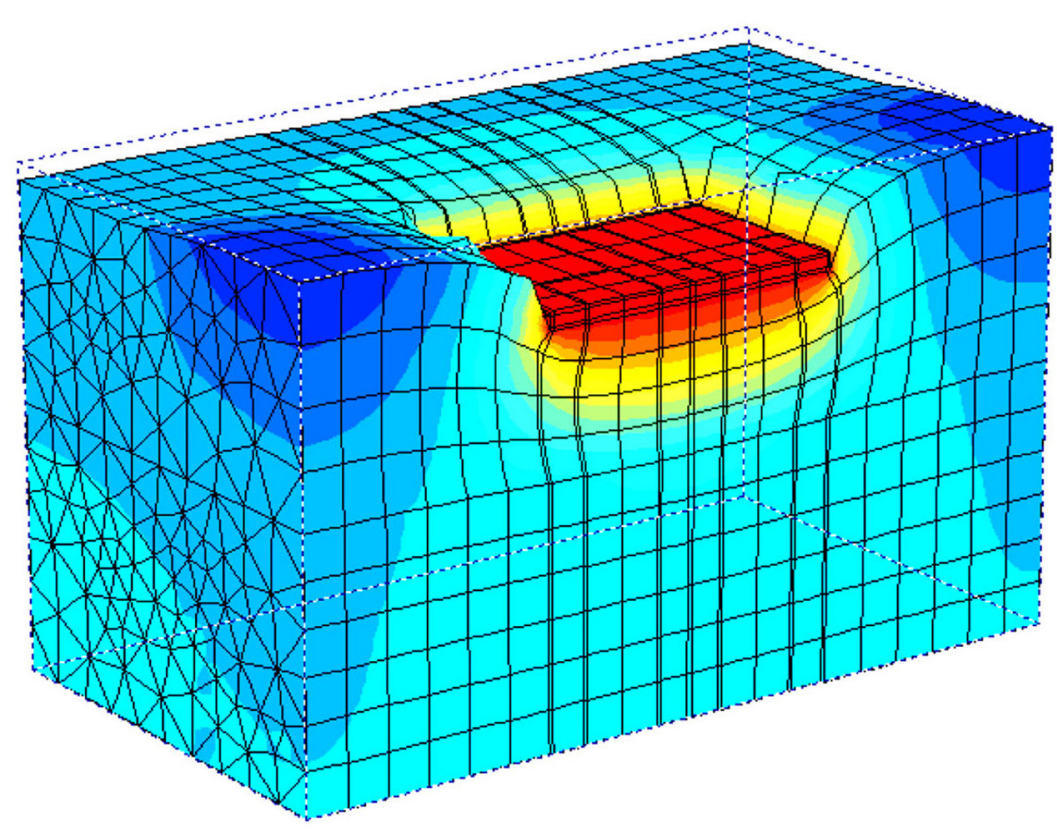

$[\times 10 \cdot 3 \mathrm{~m}]$

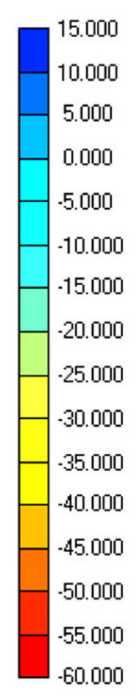

Figura 14. Deformaciones obtenidas en Plaxis para la losa maciza. 


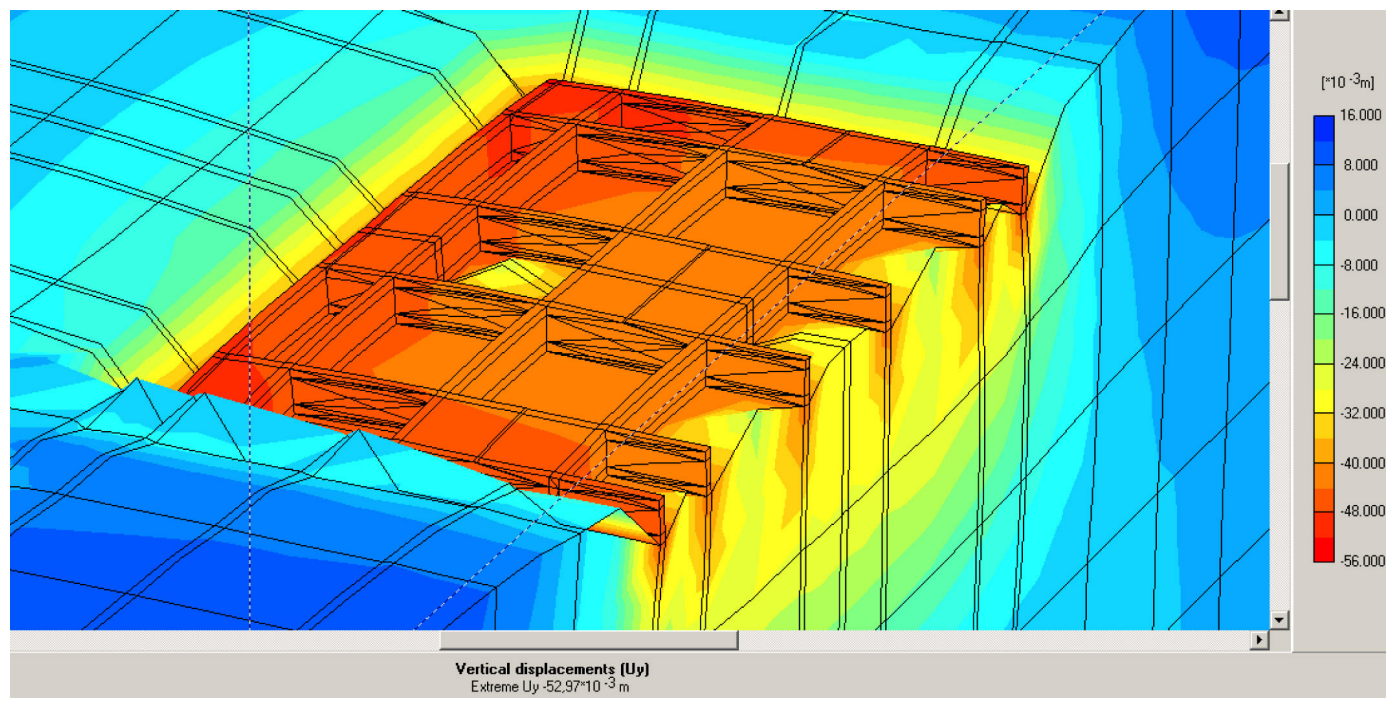

Figura 15. Deformaciones obtenidas en Plaxis para la solución zapata - emparrillado.

colocación de separadores en esa zona. Además esta solución también dificultaría el hormigonado. En contrapartida, el sistema planteado supone un ahorro importante en cuanto al volumen de hormigón, en particular en los dos casos que se han analizado, este ahorro supone un $36 \%$ de ahorro para la losa menor, y un $45 \%$ en el caso de la mayor. Como es lógico estos valores solo son orientativos, ya que en cada caso particular las dimensiones de las zonas macizas, ancho de nervios y cantos variarán, y deberán estudiarse adecuadamente.

En cuanto al proceso de ejecución de la losa propuesta, se podrían establecer dos alternativas: la primera de ellas consis- tiría en un hormigonado completo del elemento en una sola fase, lo que podría quizás plantear problemas de penetración del hormigón bajo los bloques de EPS, dado el pequeño espesor de la placa inferior. La otra opción sería un hormigonado por fases, en el que inicialmente se hormigona únicamente la placa inferior y posteriormente el resto del elemento, con la consiguiente repercusión en los plazos de ejecución. En caso de optarse por la primera de las alternativas, sería necesario disponer una especie de «pates» que mantengan los bloques separados de la armadura de la placa inferior; siendo, en cualquier caso, conveniente disponer un sistema (podrían ser barras en forma de U invertida, dispuestas entre los bloques)
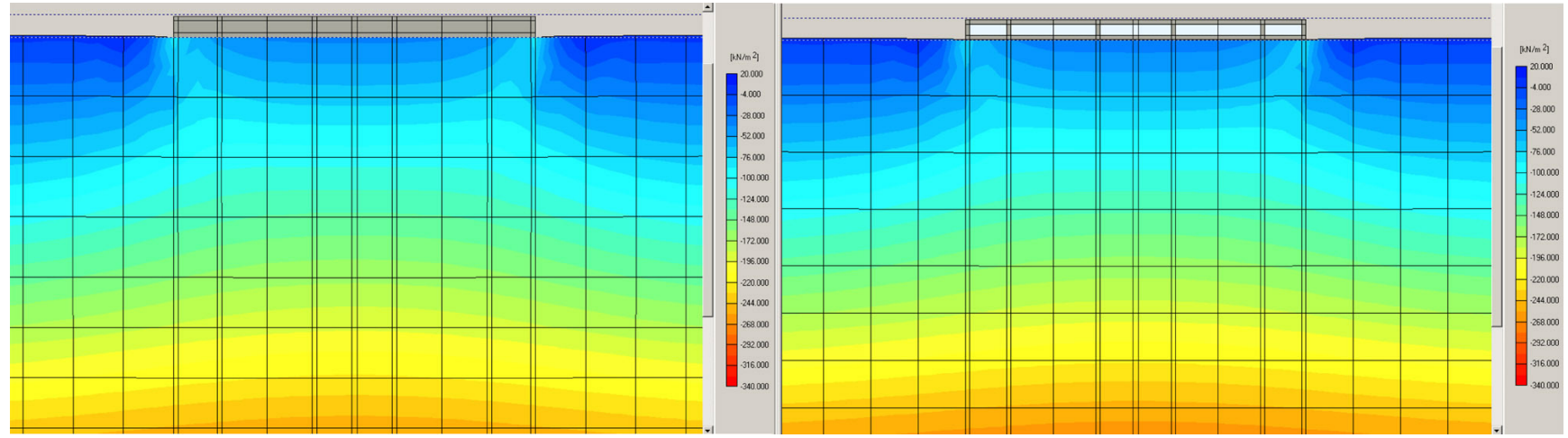

Figura 16. Resultado de tensiones verticales. Sección longitudinal por el eje de simetría.
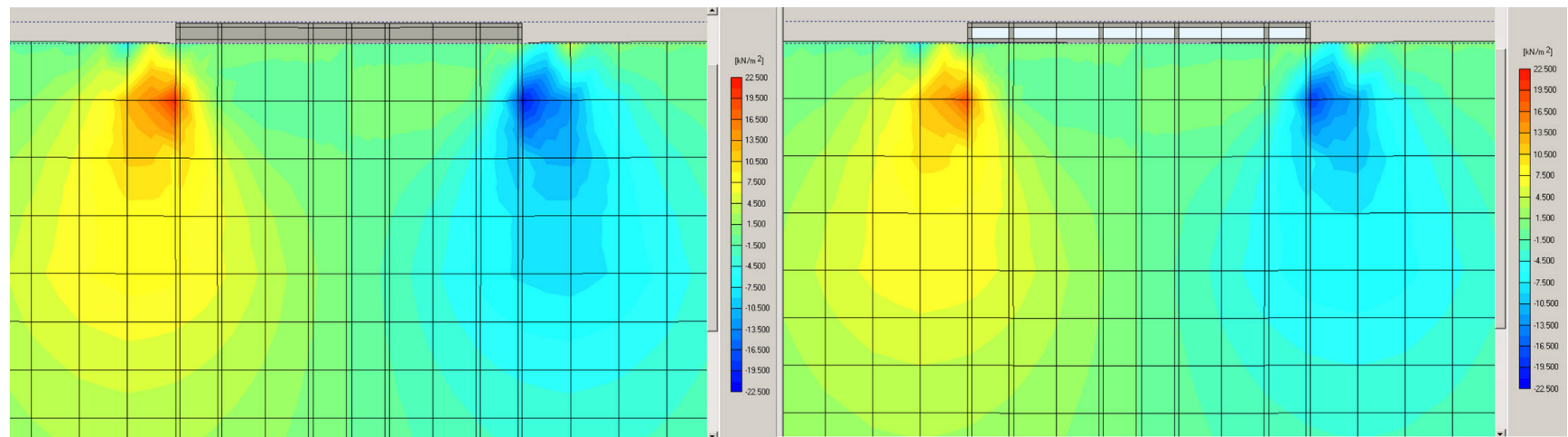

Figura 17. Resultado de tensiones de corte. Sección longitudinal por el eje de simetría. 
para evitar su desplazamiento lateral durante el hormigonado. Un sistema similar al de la placa inferior, sería necesario para colocar la armadura de la placa superior.

\section{CONCLUSIONES}

Se ha llevado a cabo un análisis comparativo de una cimentación mediante losa maciza frente a una que presenta zonas macizas bajo los pilares, unidas entre sí por nervios, todo ello junto a una losa inferior y otra superior.

Para el análisis se emplearon diferentes modelos en cuanto a las condiciones de carga, geometría y condiciones de apoyo; utilizando distintas aplicaciones informáticas.

Con los resultados obtenidos (resumidos en la Tabla 8), podríamos concluir con los puntos siguientes:

- Al emplear la solución propuesta de losa aligerada se obtienen importantes ventajas: se reducen de manera significativa los asientos de la cimentación, junto a una reducción, así mismo, de los términos diferenciales entre distintos puntos de la misma. Junto a esto, se obtiene una reducción de importancia en el volumen de hormigón y las cuantías de acero.

- Las ventajas antes expuestas no pueden extenderse de forma general, sino que, cada caso particular debe ser detenidamente analizado, ya que en la bondad de la solución influyen diversos factores, como son las condiciones de carga y geometría, como aspectos fundamentales. Las condiciones particulares del terreno de apoyo, como no puede ser de otra forma, influyen también, pero más que en la bondad en sí de la solución, lo harían en la posible rentabilidad de la misma.

- Se ha podido comprobar así mismo, que la solución es más efectiva para cargas de cierta importancia y luces también grandes, donde los problemas de asiento pueden ser más relevantes.

- Se puede afirmar también, que esta solución presentaría ventajas en el caso de edificios de dos a cuatro plantas en terrenos blandos, siendo más difícil su empleo en el caso de edificaciones de mayor altura.

- Pese a las claras ventajas que presenta la propuesta, se debe también aclarar, que su empleo dificultaría en cierta forma la ejecución.

Tabla 8. Resultados más relevantes obtenidos (acciones características).

\begin{tabular}{|c|c|c|c|c|c|c|c|c|}
\hline \multirow{2}{*}{$\begin{array}{c}\mathbf{K}\left(\mathbf{k N} / \mathbf{m}^{\mathbf{3}}\right) \\
\begin{array}{c}\text { ASIENTOS } \\
(\mathrm{mm} .)\end{array}\end{array}$} & \multicolumn{4}{|c|}{$\begin{array}{c}\text { LOSA 8,10 × 6,45 } \\
\left(K_{\text {losa }}=2.000\right)\end{array}$} & \multicolumn{4}{|c|}{$\begin{array}{l}\text { LOSA } 15 \times 12 \\
\left(K_{\text {losa }}=2.000\right)\end{array}$} \\
\hline & $\mathrm{S}_{\max }$ & $\mathrm{S}_{\min }$ & $\mathrm{S}_{\text {medio }}$ & $\begin{array}{l}\text { Diferencia } \\
\mathrm{S}_{\max ,} \mathrm{S}_{\min }(\%)\end{array}$ & $\mathrm{S}_{\max }$ & $\mathrm{S}_{\min }$ & $\mathrm{S}_{\text {medio }}$ & $\begin{array}{l}\text { Diferencia } \\
\mathrm{S}_{\max } \mathrm{S}_{\min }(\%)\end{array}$ \\
\hline MACIZA & 25,7 & 23,9 & 24,8 & 7,0 & 32,4 & 24,6 & 28,5 & 24,1 \\
\hline ALIGERADA & 24,9 & 24,2 & 24,6 & 2,8 & 23,9 & 20,9 & 22,4 & 12,6 \\
\hline
\end{tabular}

\section{REFERENCIAS}

(1) Espel, R., Gomez, J., Grima, R., Aguado, A. (2009). La evolución de la construcción del Templo de la Sagrada Familia. Informes de la construcción, 61(516): 5-20, doi: http://dx.doi.org/10.3989/ic.08.057.

(2) Lin, L.K., Chen, L.H., Chen, R.H.L. (2010): Evaluation of Geofoam as a Geotechnical Construction Material. Journal of Materials in Civil Engineering, 22(2): 160-170, doi: http://dx.doi.org/10.1061/(ASCE)o899-1561(2010)22:2(160).

(3) Negussey, D., Huang, X. (2006). Modulus of Subgrade Reaction for EPS Geofoam. En Proceedings of GeoShanghai International Conference 2006, (pp. 165-172). Shanghai. Doi: http://dx.doi.org/10.1061/40866(198)21.

(4) Miao, L., Wang, F., Han, J., Lv, W., Li, J. (2013). Properties and Applications of Cement-Treated Sand-Expanded Polystyrene Bead Lightweight Fill. Journal of Materials in Civil Engineering 25(1): 86-93, doi: http://dx.doi.org/10.1061/ (ASCE)MT.1943-5533.0000556.

(5) Braja, M. Das. (2010). Principles of Foundation Engineering (7 ${ }^{\mathrm{a}}$ edición). Cap. 5. Boston, MA (USA): Cengage Learning.

(6) Qiu, B. Li, Z. (2007). Discussion on design method of raft foundation. Shanxi Architecture 2007.

(7) Torrijo, F. J. (2010). Cimentaciones: alternativas de formulación al CTE, (pp. 11-15). Barcelona: Colegio Oficial de Geólogos - Delegación de Cataluña.

(8) Grupo Forel. http://www.forel.es/sistema_constructivo_cimentur.php. Sistema Cimentur, cimentaciones aligeradas.

(9) Valdbjørn, T. (2010). Integrated Strip Foundation Systems for Small Residential Buildings. The Open Construction and Building Technology Journal, 4: 39-53.

(10) Negussey, D. (2007). Design parameters for EPS geofoam. Soils and Foundations. Japanese Geotechnical Society, 47(1): 161-170, doi: http://dx.doi.org/10.3208/sandf.47.161.

(11) Wang, Z., Liu, Y., Sun, J., Li, C. (2007). The new super element on the interactional analysis of frame structure and Winkler foundation beams. Sichuan Building Science.

(12) Ranjan, R., Sekar, P., Krishnan, P. (2011): Raft foundation on flexffile base for compact transmission line. Power Engineer Journal, 13(2): 19-24.

(13) He, C., Cai, J. (2006): Grillage Beams on elastic foundation analized by a semi-analytical and semi-numerical method. Chinese Journal of Geotechnical Engineering, 28(6): 761-766. 